\title{
Aproximaciones teóricas al régimen del dolor en el cuerpo de las mujeres*
}

\author{
Rocío Alorda Zelada** \\ Universidad de Chile \\ rocio.alorda.zelada@gmail.com
}

El Movimiento Pro-Emancipación de la Mujer Chilena, MEMCH, posee una correspondencia inédita en donde las mujeres relatan la construcción del movimiento feminista entre los años 1935 y 1940 . Además de narrar ese proceso político, las cartas se transforman en soportes físicos de sus dolencias y enfermedades. El régimen del dolor y la articulación del movimiento feminista más grande Chile se materializa en esa correspondencia, en donde cuerpo y feminismo construyen el espacio político de las mujeres. La construcción teórica de ese cuerpo político y el régimen del dolor es lo que indaga este artículo, en donde se revisarán sus principales propuestas teóricas a partir de una mirada biopolítica del cuerpo. Este ensayo constituye un paso preliminar para encontrar los puntos de fuga en donde el dolor se moviliza y se convierte en acción política y/o feminismo.

Palabras clave: Cuerpo, dolor, enfermedad, régimen del dolor, feminismo, movimiento de mujeres.

The Chilean Women's Emancipation Movement, $\mathrm{MEMCH}$, has an unpublished correspondence where women narrate the construction of the feminist movement between 1935 and 1940. In addition to outlining this political process, the letters are transformed into a physical support of their ailments and diseases. The pain regime and the articulation of the largest feminist movement in Chile are materialized in that correspondence, where the body and feminism build the political space for women. This article investigates the theoretical construction of that political body and the pain regime, reviewing main theoretical proposals from a biopolitical perspective of the body. This essay is a preliminary step to finding the vanishing points where pain is mobilized and converted into political action and/or Feminism.

Keywords: Body, Pain, Sickness, Pain Regime, Feminism, Women's Movement

Recibido: 18 de marzo de 2013

Aceptado: 7 de agosto de 2013

\footnotetext{
* Estas reflexiones son parte del proyecto de tesis titulado "Régimen del dolor y feminismo: Prácticas políticas y estrategias de emancipación en el cuerpo adolorido de las mujeres MEMCH" para optar al grado de Magister en Comunicación Política en la Universidad de Chile. ** La autora es Periodista de la Universidad de Santiago de Chile, candidata a Magister en Comunicación Política de la Universidad de Chile y tesista Fondecyt. Sus líneas de investigación son: teoría feminista, movimiento de mujeres, comunicación política.
} 


\section{Introducción}

Una extensa correspondencia del Movimiento Pro-Emancipación de la Mujer Chilena, MEMCH, escrita entre 1935 y 1940, reúne las experiencias de mujeres de diversos rincones de Chile, quienes narran con tinta el proceso de construcción del movimiento femenino más grande que Chile haya tenido hasta la fecha. Más de 500 cartas conforman este archivo de material inédito, que más que un registro burocrático de la administración del movimiento, es un espacio íntimo de diálogo entre las mujeres de regiones y la mítica figura de Elena Caffarena, feminista ampliamente conocida por sus luchas en materia de derechos civiles y por liderar durante años el MEMCH.

Pero las mujeres mediante dichas correspondencias no solo narran su experiencia en la construcción del movimiento feminista, sino que además se quejan de infinitos dolores que tratan de fugarse por el "feminismo" en construcción. Ese registro contempla el reflejo de cuerpos femeninos adoloridos, quejumbrosos, de cuerpos incómodos en sí mismo, de la enfermedad y de la urgencia en generar vías de escape frente al dolor. Los quejidos de las mujeres de principios del siglo XX siguen vivos en esas correspondencias.

Explorar ese cuerpo adolorido de las mujeres fue el punto de partida para realizar esta investigación, que es parte del proyecto Fondecyt ${ }^{1}$ que aborda el tema del género y el dolor en las correspondencias del MEMCH y de Gabriela Mistral. La propuesta fue localizarnos en el abundante material del MEMCH y arraigar el cuerpo bajo una concepción biopolítica. De ahí que la investigación base de este artículo se titule "Régimen del dolor y feminismo: prácticas políticas y estrategias de emancipación en el cuerpo adolorido de las mujeres $\mathrm{MEMCH}^{\prime \prime}$.

Cómo se configura el régimen político del dolor en el contexto moderno focalizándolo en el cuerpo de las mujeres y cuál ha sido el desarrollo teórico de los paradigmas que alojan ese régimen, es nuestra pregunta inicial, ya que aquí queremos explorar de qué forma los regímenes políticos del dolor se configuran en los cuerpos colectivos e individuales de las mujeres; analizando -en este primer momento- sus principales desarrollos teóricos.

Sabemos que el cuerpo político de las mujeres se ha configurado, principalmente, como un espacio hegemónico, de poder y control, marcadamente influenciado por los dispositivos del capitalismo moderno, en donde además convergen prácticas culturales, políticas, económicas, performáticas, lingüísticas, materiales, tecnológicas, etc. Bajo estos acuerdos se pensará el régimen del dolor y el cuerpo de las mujeres como reflejos de procesos políticos, económicos y culturales de los momentos históricos, en donde la configuración del género pautea y promueve la construcción de un cuerpo enfermo y adolorido, como clave biopolítica que ratifica a ese territorio como el lugar depositario del gobierno de la vida. Si el cuerpo es el lugar de despliegue de la vida -según

1 Proyecto Fondecyt N 1120098 "Género y dolor: prácticas de emancipación en correspondencias de Elena Caffarena y de Gabriela Mistral durante la primera mitad del siglo XX". Investigadora responsable: Diamela Eltit; Coinvestigadora, Eugenia Brito; tesista, Rocío Alorda. 
Foucault-, el cuerpo femenino será el lugar destinado para la representación material o simbólica del dolor, de la amenaza y la enfermedad.

Lo que aquí queremos hacer -como dice Diamela Eltit- es oír el dolor, "escuchar el dolor que nos parezca más próximo y más político. Establecer una política para escuchar el dolor y la enfermedad" (Eltit en CUDS, 2010) y revisar sus territorios.

A continuación presentamos las primeras aproximaciones teóricas a esa discusión sobre el régimen del dolor en el cuerpo de las mujeres.

\section{Discusión bibliográfica}

\section{Las marcas de los cuerpos}

El cuerpo, como zona primordial de anclaje de los discursos sociales, políticos y culturales, es un espacio privilegiado para pensar cómo las redes de dominación escriben y describen el rol de la mujer, tanto en su acontecer simbólico como también su desplazamiento e intervención en el espacio público. En ese sentido, el cuerpo como dispositivo de vinculación con el mundo se relaciona mediante actividades perceptivas, emocionales, de apariencia y de relación con el sufrimiento y el dolor.

Lo que se pone en juego en el cuerpo es una serie de sistemas simbólicos y significaciones que van a constituir la base de las experiencias individuales y colectivas. De las diversas significaciones que se les da al cuerpo, una de las principales es la condición ficcional de este, que opera por medio de la cultura, la que dibuja las conductas e imaginarios corporales en un tiempo y lugar definido (Le Breton, 2002). Así, la construcción social del cuerpo no es solo la suma de las relaciones entre el mundo y la corporeidad, sino también en la determinación de su naturaleza, en donde el cuerpo no existe en el estado natural, sino que "siempre está inserto en la trama del sentido, inclusive en sus manifestaciones aparentes de rebelión, cuando se establece provisoriamente una ruptura en la transparencia de la relación física con el mundo del actor (dolor, enfermedad, comportamiento no habitual), etc." (Le Breton, 2002: 33).

En ese sentido, el cuerpo como construcción cultural es un diseño de tal magnitud que desplaza la biología y la somete a la cultura, es decir, al discurso. El cuerpo, así, cambia, se desorganiza o se reorganiza de acuerdo con dictámenes que provienen de la suma de economías que ordenan la totalidad del sistema social ${ }^{2}$. El cuerpo, como lugar de anclaje de discursos, es además el territorio en donde se junta la vida y la administración jurídica de esta. Así, es preciso reconocer que el modo en que los cuerpos de las mujeres de principios del siglo pasado fueron configurados -en un contexto de desarrollo

2 Marco teórico proyecto Fondecyt, "Género y dolor: prácticas de emancipación en correspondencias de Elena Caffarena y de Gabriela Mistral durante la primera mitad del siglo XX". 
de la industrialización capitalista-, el sistema de dominación de los cuerpos ya había constituido un primer anclaje desde una perspectiva biopolítica.

De ahí la importancia de fijar la mirada en el desarrollo teórico de la biopolítica como "la implicación cada vez más intensa y directa que, a partir de una cierta fase ubicable en la segunda modernidad, viene a determinarse entre las dinámicas políticas y la vida humana, entendida en su dimensión específicamente biológica" (Esposito, 2009:126). El enfoque biopolítico del cuerpo permite arraigar la diversidad de discursos de la época para comprender sus configuraciones y tensiones.

Estos cuerpos de mujeres con huellas biopolíticas hablan de diversas formas en el siglo pasado. María Angélica Illanes (2003), quien desarrolla un análisis sobre la construcción de los cuerpos populares chilenos a fines del siglo XIX y principios del siglo XX, se focaliza en la categoría cuerpo y sangre abordándolo a partir de cuatro perspectivas: como materialidad biológico-social, como corpus biocientífico portador de ética y verdad, como vía de aproximación al pueblo y desde la perspectiva del movimiento obrero (Illanes, 2003). La perspectiva de Illanes permite comprender la configuración del espacio social en la primera mitad del siglo XX, para conocer el cuerpo enfermo, adolorido y amenazado de las mujeres de los sectores populares; cuerpos y discursos que se ven retratados en las cartas que las mujeres del MEMCH escribían.

Sin embargo, a finales del siglo XX y particularmente el siglo XXI, cambia el paradigma. El detallado énfasis en los matices del cuerpo va a ser erradicado por lo saludable, asociado a la juventud como patrimonio en la actual época postindustrial, marcada por el consumo y la masiva inserción en la flexibilidad laboral y las labores de prestación de servicios. Esa queja femenina que fuera legitimada por su época, hoy ha desaparecido del escenario social, ya que la queja ha sido anestesiada. Pero para entender cómo el dolor orgánico ha sido reprimido y modificado por el consumo de salud y juventud (gimnasio, pabellones quirúrgicos, consumos estéticos) nos parece necesario entender cómo funcionó el cuerpo-dolor en el siglo $\mathrm{XX}^{3}$.

\section{Cuerpo de las mujeres, cuerpo político del femenino}

Las vinculaciones entre cuerpo y política ha sido una preocupación constante dentro del feminismo, el que estalló justamente -como señala Marta Lamas-

a partir de la interpretación que se hacía de la diferencia entre los cuerpos de los hombres y las mujeres: puesto que tenían anatomías distintas, sus capacidades intelectuales y sus papeles sociales también habían de diferir; así, se aceptaba tranquilamente que las mujeres no tuvieran los

3 Marco teórico proyecto Fondecyt, "Género y dolor: prácticas de emancipación en correspondencias de Elena Caffarena y de Gabriela Mistral durante la primera mitad del siglo XX". 
mismos derechos ni las mismas opciones de vida que los hombres (Lamas, 1994: 3).

La teoría feminista en las últimas tres décadas ha generado una diversidad de reflexiones a partir del cuerpo, su construcción, administración e influencia cultural: desde el estudio relativo a las representaciones sociales hasta la definición de las políticas específicas sobre la reglamentación del uso sexual y reproductivo del cuerpo. "La tendencia intelectual dominante trabaja sobre la construcción social del cuerpo: desde la forma en que culturalmente se otorgan nuevos significados a diversos aspectos corporales, hasta las reformulaciones políticas que controlan y regulan diferencialmente los cuerpos" (Lamas, 1994: 7).

El aporte del feminismo ha sido colocar el cuerpo en la agenda pública analizando la construcción del sujeto sin dejar afuera la materialidad de la diferencia sexual. Sin embargo, sabemos que los cuerpos femeninos -individuales y colectivos- no son territorios neutrales, sino que, muy por el contrario, en ellos convergen una serie de dispositivos que lo marcan y limitan a través de una diversidad de expresiones.

En el cuerpo, el género funciona como esquemas mentales y corporales de percepción que lo conforma y lo engendra y que está inmerso en el orden social, tratándose de "una institución que ha estado inscrita por milenios en la objetividad de las estructuras sociales y en la subjetividad de las estructuras mentales (...) estos establecidos como conjuntos objetivos de referencias, los conceptos de género estructuran la percepción y la organización concreta y simbólica de toda la vida social" (Bourdieu, 1986: 91).

Más allá de la construcción binaria del cuerpo a base de la lógica heteronormativa, teóricas feministas han planteado la deconstrucción del género como un proceso de "subversión cultural". Tal es el caso de Judith Butler, quien concibe el género como "el resultado de un proceso mediante el cual las personas recibimos significados culturales, pero también los innovamos" (Butler, 2007) a base de una estrategia de desnaturalización de los cuerpos y resignificación de las categorías corporales. Para Butler, la construcción del sexo es una práctica reiterada alojada en matrices de relaciones. "La construcción no solo se realiza en el tiempo, sino que es en sí mismo un proceso temporal que opera a través de la reiteración de normas, en el curso de esta reiteración el sexo se produce y a la vez se desestabiliza" (Butler, 2002: 29). Mientras tanto, Donna Haraway (1995) cuestiona la noción de identidad de género y las distinciones entre sexo y género, categorías conceptualizadas por el feminismo pero que a su vez han tenido nociones no examinadas del sexo, el cuerpo, la naturaleza y la biología. Los límites que estas categorías instalan se han reflejado en los discursos feministas a base de la ilusión de una "unidad común entre todas las mujeres" (Haraway, 1995: 37) y se ha ignorado que la "naturaleza" de las mujeres está estructurada por las historias de la dominación.

Esos cuerpos femeninos a lo largo de la historia humana han pasado por procesos de disciplinamiento y de instalación de técnicas de poder, transformándose en un territorio clave para entender el origen del dominio masculino 
y de su construcción identitaria que ha sido instrumentalizada para la consolidación del poder patriarcal y la explotación de la fuerza de trabajo de las mujeres (Federici: 2004). Esta idea desarrollada por Silvia Federici arraiga la noción de que en el cuerpo femenino no solo se congregan técnicas de dominación, sino que también relaciones de poder conectadas a partir de los contextos sociales y económicos. De ahí la importancia de documentar las condiciones bajo los cuales los cuerpos femeninos se configuran, ya que en la sociedad capitalista

el cuerpo es para las mujeres lo que la fábrica es para los trabajadores asalariados varones: el principal terreno de su explotación y resistencia, en la misma medida en que el cuerpo femenino ha sido apropiado por el Estado y los hombres, forzado a funcionar como un medio para la reproducción y la acumulación de trabajo. En este sentido, es bien merecida la importancia que ha adquirido el cuerpo, en todos sus aspectos -maternidad, parto, sexualidad-, tanto dentro de la teoría feminista como en la historia de las mujeres (Federici: 2004: 28-29).

En ese contexto público en el que se despliega la corporalidad, hablamos de un "régimen político del cuerpo", en tanto territorio principal de las políticas sociales y económicas capitalistas. Silvia Federici nos dice: "la primera máquina desarrollada por el capitalismo fue el cuerpo humano y no la máquina de vapor, ni tampoco el reloj" (Federici, 2004: 201).

\section{El cuerpo como huella biopolítica}

La producción de conocimiento generada en la segunda mitad del siglo XX trajo consigo nuevos campos epistemológicos que han permitido situar de mejor forma a la política, su relación con la vida, el cuerpo y el poder. Bajo ese marco, Foucault desarrolla el concepto de biopolítica que "se refiere a la implicación cada vez más intensa y directa que se establece, a partir de cierta fase que se puede situar en la segunda modernidad, entre las dinámicas políticas y la vida humana entendida en su dimensión específicamente biológica" (Esposito, 2005: 7).

La biopolítica en tanto mixtura de lenguajes, explica la politización de la vida y la biologización de la política, donde la vida se instala en el centro del juego político al igual que las prácticas de muerte. La biopolítica intenta comprender el complejo proceso de "disciplinamiento del cuerpo que generó las condiciones para el desarrollo del capitalismo en un intento por parte del Estado y de la Iglesia por transformar las potencias del individuo en fuerza de trabajo" (Federici, 2004: 179) a través de técnicas de control social materializadas, principalmente, en la preocupación estatal frente al control sanitario y sexual de los cuerpos.

Lo que hace Foucault es comprobar que las sociedades occidentales funcionan como sociedades disciplinarias, en donde sus miembros están adscritos a mallas cerradas que controlan sus movimientos. Esta disciplina dibuja otro tipo de relaciones, más allá de las instaladas por el Estado, que 
se transforma en un ejercicio de poder que atraviesa diversas instituciones convergiendo en un sistema de sumisión y eficacia. Le Breton lo explica de la siguiente forma:

Foucault desplazó los parámetros de análisis que en ese momento gozaban de mayor favor y atrajo la atención sobre las modalidades eficaces y difusas del poder cuando se ejerce sobre los cuerpos que están más allá de las instancias oficiales del Estado (..). El campo político que se esfuerza por organizar las modalidades de acuerdo con las finalidades propias refiere a una tecnología puntillosa del cuerpo, una política del detalle, más que una confiscación sin medida de un Estado, medio de dominación de las clases dominantes (Le Breton, 2002: 84).

Así, Foucault llega a la conocida conclusión de que el poder se ejerce más que se posee, ya que no es un privilegio de una clase dominante, sino que "el efecto conjunto de sus posiciones estratégicas", donde sus efectos de dominación son atribuidos a dispositivos que logran normalizar los cuerpos individuales y colectivos.

Sin embargo, si el cuerpo es el territorio en donde se junta la vida y la administración jurídica de esta, en el contexto moderno, la vida no puede desarrollarse de cualquier manera. Para determinar el modo en que la vida avanza está la "condición inmunológica", paradigma desarrollado por Roberto Esposito que será definido como la tendencia a proteger la vida desde las amenazas externas en el cuerpo individual como colectivo.

Un verdadero salto de calidad, en dirección mortífera, se tiene cuando este pliegue inmunitario del recorrido biopolítico se entrecruza, primero, con la parábola del nacionalismo y, luego, del racismo. Entonces, la cuestión de la conservación de la vida pasa del plano individual, típico de la fase moderna, al del Estado nacional y de la población en cuanto cuerpo étnicamente definido en una modalidad que los contrapone, respectivamente, a otros Estados y a otras poblaciones (Esposito, 2005:10).

\section{El régimen del cuerpo y sus límites: el dolor}

Desde esta perspectiva es necesario poner a salvo la vida del individuo y de la especie, y para esto la política debe conducirla al régimen del cuerpo, ya que política y vida debe considerar un tercer elemento clave que es la dimensión del cuerpo en donde se conserva la vida por la inmunización política (Esposito, 2005). Si el cuerpo es el territorio de desarrollo de la vida, también es ahí donde hay mayor riesgo de enfermedad y muerte, dicotomía que lo plantea como una zona de límite en donde en su interior se ejerce la intensión inmunitaria de la política: "Demorar cuanto más se pueda el paso de la vida a la muerte, empujar la muerte al punto más alejado de la actualidad de la vida. El cuerpo es a la vez el campo y el instrumento de este combate. Mientras resista, no habrá muerte" (Esposito, 2005: 161). En 
este régimen del cuerpo frente a sus límites, el vocabulario de la medicina y de la inmunología generan valoraciones con efectos simbólicos importantes, como lo son las enfermedades. Es la fisiología una de las principales figuras dominantes que se usan en la defensa y conservación de un cuerpo político (Ronell, 2012).

En este régimen del cuerpo, la enfermedad aparece como el punto de cruce entre el saber político y el saber médico, en la lucha por la conservación de la vida. Vida y muerte, salud y enfermedad se definen a sí mismos como opuestos y no existe uno sin el otro. De este modo, la localización de la enfermedad estará generada por dos condiciones: el colapso del cuerpo político por su envejecimiento natural y/o por una conmoción violenta. Ambos casos son males endógenos generados dentro del organismo político. Sin embargo, también existen amenazas externas que atacan al cuerpo político que se produce por el contagio de un cuerpo extraño, como una invasión extranjera, conflictos civiles, contactos con culturas desconocidas, etc. $Y$ el efecto inmediato es la necesidad urgente de cerrar toda frontera que posibilite el acceso al ente amenazador, es decir, comienzan a funcionar los mecanismos de autodefensa. No obstante, la feroz amenaza tiene su fin en sí mismo, ya que

si la cura contra el veneno está en el veneno mismo, entonces enfermedad y salud ya no se dispondrán a lo largo del eje de una contraposición frontal, sino según una relación que hace de una el contrario, sobre todo al instrumento de la otra (..) allí donde hay enfermedad, hay medicina; allí donde hay medicina, hay enfermedad (Esposito, 2005:178).

Foucault relacionó la crisis de la soberanía y la biopolítica como dos instancias antagónicas, ya que mientras la soberanía ejerce su régimen a través del derecho a dar muerte, la biopolítica es la perspectiva del cuidado de la vida. Sin embargo, como ya hemos vitos, no existe vida sin muerte y bajo esa relación el cuerpo es el elemento unificador del sujeto colectivo e individual. Por eso, explica Esposito (2005), el saber médico a partir del siglo XVIII irá tomando una mayor relevancia al interior de la política, ya que cuando el cuerpo de los ciudadanos pasa a ser un lugar de ejercicio del poder el tema de la salud pública será uno de los elementos centrales bajo los cuales funcionarán la actividad política, económica y administrativa del Estado.

Con todos estos dispositivos operando, el cuerpo se convierte en un objeto de biopoder que para Donna Haraway (1995) tiende a deconstruir su caracterización unitaria poniendo el cuerpo al centro de las tecnologías, electrónicas e informáticas. "La relación entre política y vida ahora pasa por un filtro biotecnológico que descompone ambos términos antes de volver a asociarlos en una combinación, material y figurada, inasible para el aparato foucaultiano" (Esposito, 2005: 207). Haraway no se aleja del horizonte biopolítico, sino que pone el nexo entre vida y política en un contexto de proliferación de la tecnología. En palabras de Haraway, "el sistema inmunitario es un plan para una acción encaminada a la construcción y al mantenimiento de los límites 
de los que cuenta como sí mismo y como otro en los ámbitos cruciales de lo normal y de lo patológico" (Haraway, 1995:137). Así bajo ese régimen los cuerpos modernos no nacen, sino que se construyen "desnaturalizados como signo, contexto y tiempo". Los organismos son fabricados en un mundo que cambia constantemente. El paradigma immunitario avanza un paso más que el paradigma biopolítico, ya que se configura como el lugar neurálgico por donde pasa el gobierno político de la vida.

\section{El régimen del dolor}

En las sociedades modernas la democracia es entendida principalmente como un sistema de inmunidad, que se establece como una norma general o un sistema de garantías del que se benefician las personas, los cuerpos y las opiniones. No obstante, la vida en comunidad supone un riesgo común, algo antagónico al sistema inmunitario (Brossat, 2008). Frente a la dicotomía que genera la democracia bajo el paradigma inmunitario se producen mecanismos o técnicas de "anestesia" que se desarrollan a mediados del siglo XIX en la búsqueda de producir la insensibilidad de una zona. Estos mecanismos anestésico en la época moderna ya no tratan solamente de disminuir el dolor, sino de crear estados de insensibilidad a través de desarrolladas técnicas que pueden generar efectos programados en la búsqueda de acrecentar el paradigma civilizatorio basada en la "supresión masiva del dolor vinculada a las enfermedades o a los accidentes que acompañan los cuidados y las operaciones, al punto de sentir en común algo como un derecho a no sufrir" (Brossat, 2008:57).

En este contexto anestésico, el régimen del cuerpo se caracteriza por su indolencia a través de un tratamiento específico al dolor, elemento vinculado fuertemente a la dignidad, en donde el no dolor será percibido como algo civilizado y el sufrimiento será la decadencia. Así, el dolor se configura, en un primer momento, como un horizonte insuperable de la condición humana. Nadie puede huir de él, ya que ha surgido tal y cual como la muerte. Más allá de que el dolor sea un mal, es también configurado como un "medio de fortalecer moralmente", a través de prácticas de resistencia y domesticación del dolor, la lucha entre el "hombre" y el sufrimiento, la valentía viril y el control de sí (Brossat, 2008).

De este modo el dolor será visto por la modernidad como un mal absoluto, en palabras de Hannah Arendt, será una absoluta no pertenencia al mundo (Arendt en Brossat, 1981: 225) y bajo este contexto aparecerá la noción de "crimen contra la humanidad", en donde además se incluyen los actos de tortura y violencia y dolor extremo. Asimismo se rechaza el dolor y la violencia como anomalías, en donde la condición anestésica aparece como una pacificación y calma al dolor con el entorno o con el organismo, en una forma de autocontrol del cuerpo que se enfrenta a la dolencia. "La contraparte del distanciamiento del dolor es la dependencia de nuestras existencias frente a procedimientos, a sustancias y también a potencias que disponen de medios para producir anestesia. Es bajo las condiciones del poder del médico y de los poderes públicos que lo autoriza, que nos emancipamos del dolor" (Brossat, 2008: 80). 
Así, la anestesia al dolor, como dispositivo biopolítico, se incorpora en la sociedad moderna con distintas materialidades: el sedante, la morfina, las terapias alternativas, hasta dispositivos más sofisticados en donde los propios medios de comunicación pueden funcionar como anestesiadores del cuerpo político.

Los cuerpos que más sufren serán entonces los que estén destinados a la normalización de ese dolor, como vía elemental del proyecto emancipador en la modernidad. Sin embargo, esos cuerpos dolientes se ven enfrentados a metáforas -como las que generan las enfermedades- que también se materializan en cuerpos enfermos y principalmente en cuerpos femeninos.

Susan Sontag nos acerca a esa reflexión a través del análisis que genera sobre las metáforas en torno al cáncer y la tuberculosis, como huellas biopolíticas de dos momentos históricos: siglo XIX y siglo XX. Las fantasías inspiradas por la tuberculosis en el siglo XIX y por el cáncer hoy, son reacciones ante enfermedades consideradas intratables y caprichosas -es decir, enfermedades incomprendidas-, precisamente en una época en que la premisa básica de la medicina es que todas las enfermedades pueden curarse. Las enfermedades de ese tipo son, por definición, misteriosas. Porque mientras no se comprendieron las causas de la tuberculosis y las atenciones médicas fueron tan ineficaces, esta enfermedad se presentaba como el robo insidioso e implacable de una vida (Sontag, 2003).

Es interesante la relación que Sontag genera sobre el cáncer y las mujeres. Según explica, fue Galeno (siglo II d. C.) quien observó que "la mujer melancólica" era más propensa al cáncer de mama que "la mujer sanguínea", en donde "Galeno entendía por melancolía un estado fisiológico de síntomas temperamentales complejos; nosotros no entendemos más que un mero estado de ánimo". "La aflicción y el ansia", decía en 1845 sir Astley Cooper, cirujano inglés, están entre "las causas más frecuentes" de cáncer de pecho (Sontag, 2003:25). Serán entonces las condiciones emocionales que supuestamente las mujeres poseen las generadoras de este mal, que metafóricamente estaría más vinculado a una característica femenina que masculina. Aquí, los cuerpos de mujeres melancólicas estarían destinados a sufrir enfermedades como el cáncer. A criterio de Sontag, estas metáforas sobre las enfermedades, específicamente sobre el cáncer, "evoca otro tipo de catástrofe económica: la del crecimiento incontrolado, anormal, incongruente" (Sontag: 2003:30), de ahí la urgente necesidad de actuar a tiempo y sobre todo de prevenir.

\section{Reflexiones finales}

Lo realizado hasta este momento fue configurar un escenario en donde se desarrolla el régimen del cuerpo y del dolor en un contexto biopolítico en la modernidad, para acercarnos a los modos en que el cuerpo femenino se construye. Las cartas de dolor de las mujeres del MEMCH nos sirven para armar el mapa de cuerpos femeninos que habitan a inicios del siglo XX en Chile, como referencia en la configuración de cuerpos que cargan huellas biopolíticas y que narran su condición de corporalidad en sufrimiento. 
Esta reflexión teórica permite situar y comprender el desarrollo de los cuerpos en un contexto social específico, en donde dichos territorios se transforman en reflejos de un proceso de normalización de los cuerpos sociales. El cuerpo, como dispositivo de vinculación con el exterior, es el soporte adecuado para leer esos momentos históricos en los que habita, ya que ahí será el lugar de despliegue y control de la vida moderna.

De este modo, si se sitúa al cuerpo como lugar de anclaje y desarrollo del régimen moderno, revisar los discursos que ahí se instalan permite revisar sus dispositivos de control y normalización en un momento histórico en donde los cuerpos femeninos ya no son solo depositarios de las funciones reproductivas, sino máquinas de producción en un periodo de industrialización moderna. El cuerpo será el reflejo de ese proceso capitalista.

Los aportes que el feminismo entrega para mirar dicho cuerpo son de gran valor, ya que lo sitúa desde el espacio público identificando las diferencias que genera el género y los dispositivos de control que ahí se desarrollan como elemento clave de la estrategia de dominación patriarcal. Es la teoría feminista la que permite mirar el cuerpo femenino como un territorio fuertemente regulado por los discursos médicos, tecnológicos, políticos, económicos y culturales.

Sin embargo, ese cuerpo que se establece como eje del régimen político moderno, tendrá que enfrentarse a sus propios límites: la vida y la muerte, su desarrollo pleno y la enfermedad. Así, el dolor como amenaza al cuerpo civilizado no es solamente una condición biológica, sino que se constituye además como una tecnología de vulneración de ciertos cuerpos que deben ser fácilmente anestesiados. Sin embargo, la anestesia como respuesta de la sociedad moderna frente al dolor no es suficiente, ya que normalizar el sufrimiento -sobre todo en los cuerpos femeninos- no genera su emancipación.

Las búsquedas que se harán en el desarrollo de este relato será encontrar el punto de fuga de ese dolor en un complejo proceso de construcción de un movimiento político y emancipador como lo es el MEMCH. El horizonte es mirar al feminismo en construcción por las mujeres memchistas como un proceso liberador que logra superar la condición metafísica del dolor hacia destinos que sitúan el cuerpo más allá del complejo proceso normalizador que en él se han arraigado durante siglos. Es ese el camino que se transitará entre las cartas y las teorías y esta es su primera aproximación.

\section{Obras citadas}

Bourdieu, Pierre y Wacquant, Loic (1992). An invitation to Reflexive Sociology. The University of Chicago Press, p.171.

Brossat, Alain (2008). La democracia inmunitaria. Santiago: Editorial Palidonia. Butler, Judith (2002). Cuerpos que importan. Sobre los límites materiales y discursivos del "sexo". Buenos Aires: Editorial Paidós.

Butler, Judith (2007). El género en disputa. El feminismo y la subversión de la identidad. Barcelona: Editorial Paidós.

Coordinadora Universitaria por la Disidencia Sexual (2010). Por un feminismo sin mujeres. Fragmentos del Segundo Circuito Disidencia Sexual. Santiago: Editorxs. 
Esposito, Roberto (2005). Inmunitas: protección y negación de la vida.1a edición, Buenos Aires: Amorrortu Editores.

Esposito Roberto (2009). Comunidad, inmunidad y biopolítica. Barcelona: Editorial Herder.

Federici, Silvia (2004). Calibán y la bruja. Mujeres, cuerpo y acumulación original. Madrid: Edición Traficantes de Sueños.

Haraway, Donna (1995). Ciencia, cyborgs y mujeres. La reinvención de la naturaleza. Madrid: Ediciones Cátedra Instituto de Valencia, Instituto de la Mujer.

Illanes, María Angélica (2003). Cuerpo y sangre de la política. Santiago: Editorial LOM.

Le Breton, David (2002). Sociología del Cuerpo. Buenos Aires: Ediciones Nueva Visión.

Lamas, Marta (1994). Cuerpo: diferencia sexual y género, en Debate Feminista: cuerpo y política. Año 5, Vol.10 (3-29), septiembre. http:// www.debatefeminista.org

Ronell, Avital (2012). Reinas de la noche. Santiago: Editorial Palinodia.

Sontag, Susan (2003). La enfermedad y sus metáforas. Buenos Aires: Taurus Pensamiento. 